\title{
TINA und die technologische Revolution
}

\author{
Jörg Flecker ${ }^{1}$ \\ Universität Wien
}

\begin{abstract}
Wir erleben derzeit eine langanhaltende öffentliche Debatte über den Umbruch von Wirtschaft und Gesellschaft im Zuge der Digitalisierung. Dabei verbinden sich die Perspektive des Technikdeterminismus, welche die Ursache sozialer Veränderungen in der Technik sieht, und jene des Neoliberalismus. Entsprechend wird nicht die Frage gestellt, welche Technikentwicklung und -anwendung wünschenswert sei, sondern bloß eine rasche Digitalisierung zur Steigerung der Wettbewerbsfähigkeit gefordert. In diesem Beitrag wird ausgehend von aktuellen Debatten über Digitalisierung, Industrie 4.0 und Arbeit 4.0 dargestellt, welche Beschäftigungswirkungen der Digitalisierung derzeit erwartet werden und wie sich die Qualität der Arbeit abhängig von unterschiedlichen Zielsetzungen bei der Entwicklung und beim Einsatz digitaler Technologien abzeichnen. Am Beispiel der sogenannten Plattformwirtschaft wird gezeigt, wie sich die Gestaltung von Technik mit der neoliberalen gesellschaftlichen Umwälzung verbindet.
\end{abstract}

Keywords: Digitalisierung; Arbeit 4.0; Industrie 4.0; technologische Revolution

\section{Einleitung}

Seit den 1950er Jahren gab es schon mehrere hitzige, wissenschaftliche und öffentliche Debatten über die Auswirkungen der Automatisierung. „Geht uns die Arbeit aus?“ oder „Was macht dann der Mensch, und was die Maschine?" waren zentrale und wiederkehrende Fragen. Die aktuelle Diskussion über Industrie 4.0 und Digitalisierung folgt einem ähnlichen Muster. Auch diesmal werden Schreckensszenarien über den Verlust von Arbeitsplätzen von Spekulationen begleitet, ob nicht letztlich alle menschlichen Tätigkeiten im Erwerbsleben durch Roboter oder Algorithmen ausgeführt werden könnten. Etwas überraschend ist, dass diese Themen nun schon so lange kontinuierlich Aufmerksamkeit auf sich ziehen können, seit im Jahr 2011 das Schlagwort Industrie 4.0 in Deutschland geprägt worden war. Mit der Behauptung einer vierten industriellen Revolution wurde ein tiefgreifender Umbruch in der Produktion in Aussicht gestellt. Dies ging aber nicht aus einer akademischen Betrachtung der Rationalisierungsbemühungen in der Industrie hervor. Vielmehr bemühten sich die interessierten Proponenten und Proponentinnen der technologischen Innovation, technische Potenziale zur ent-

1 Jörg Flecker ist Professor für Allgemeine Soziologie am Institut für Soziologie der Universität Wien: joerg.flecker@univie.ac.at 
scheidenden Chance für den Wirtschaftsstandort zu stilisieren. Mit der verstärkten Automation auf Basis cyber-physikalischer Systeme, so hieß es, gelinge es nicht nur, die Wettbewerbsfähigkeit $\mathrm{zu}$ steigern, sondern auch Industriearbeitsplätze in die Hochlohnländer Europas zurückzuholen. Mehr noch: Dieser Umbruch wurde als ein Anliegen von nationalem Interesse dargestellt, weshalb nun alle an einem Strick ziehen und der Staat sich mit Förderungen einstellen sollte.

Zunächst handelte es sich also in erster Linie um eine Revolution in der Wahrnehmung der technischen Entwicklung in der Industrie und auch in anderen Wirtschaftssektoren: War man an große Fortschritte im Zuge der eher inkrementellen Innovation gerade unter Einsatz der Informations- und Kommunikationstechnologien, aber auch von Robotern, während der letzten Jahrzehnte gewohnt, sollte man nun einsehen, dass ein Technologiesprung bevorstand, dass disruptive Technologien dem gewohnten Gang der Dinge eine Ende setzen und ganz neue Entwicklungen auslösen würden. Der Blick wurde damit noch stärker auf die Technik gelenkt, die damit als alleinige Treiberin von Veränderungen in Organisationen, am Markt, in den Arbeitsbeziehungen und in der Politik erschien. Und die technische Entwicklung wurde als notwendig dargestellt. Die Frage war nicht, ob eine solche Entwicklung und Anwendung von Technik erwünscht ist oder nicht, sondern nur, ob es gelingt, rechtzeitig auf den Zug aufzuspringen und vorne dabei zu sein. Damit fügte sich die Debatte in das neoliberale TINA („There is no alternative“2) ein, welches die Kommerzialisierung aller Gesellschaftsbereiche sowie Wettbewerb und Wettbewerbsfähigkeit als Maximen enthält. Sie stärkte zugleich den "Standortnationalismus“ (Butterwegge et al. 1998), also die Betonung der wirtschaftlichen Konkurrenz zwischen Ländern und der Forderung nach einer darauf ausgerichteten Gesellschaft. Das Konzept von Industrie 4.0 erweist sich damit von neoliberalen Perspektiven geprägt und trägt umgekehrt zur Stärkung dieser gesellschaftspolitischen Orientierung bei (Pfeiffer 2017).

Im Einzelnen ist die Verschränkung von Technikentwicklung und -nutzung mit dem neoliberalen Programm des gesellschaftlichen Umbaus in dreierlei Hinsicht zu erkennen: Erstens wird vielfach wie selbstverständlich von einer einseitig von Unternehmensinteressen und betrieblichem Management bestimmten Gestaltung von Technik und Arbeitsorganisation ausgegangen, die eine Degradierung der Arbeit im Sinne eines neuerlichen Taylorismus oder einer Prekarisierung keineswegs ausschließt. Zweitens gehört die durch Informations- und Kommunikationstechnologie (IKT) ermöglichte Ausweitung der Optionen für eine räumliche Lösung („spatial fix“, Harvey 2001) für betriebswirtschaftliche Herausforderungen bzw. kapitalistische Krisen zum Kern der neoliberalen Globalisierung. Schließlich erfolgt drittens mit der Umsetzung neuer Geschäftsmodelle auf der Basis von Internet, Apps und mobiler Endgeräte (Stichwort: Uber) de facto eine Deregulierung und Re-Kommodifizierung von Arbeit. Alle drei Aspekte sind in der sogenannten Plattformwirtschaft besonders ausgeprägt. Sie kann damit als Spitze des Eisbergs eines umfassenden Umbruchs der Arbeitswelt gelten.

In diesem Beitrag soll ausgehend von aktuellen Debatten über Digitalisierung, Industrie 4.0 und Arbeit 4.0 untersucht werden, welche Veränderungen sich im Hinblick auf die Beschäftigung und die Qualität der Arbeit abzeichnen. Zudem wird danach gefragt, inwiefern

\footnotetext{
${ }^{2}$ Dieser Ausspruch zugunsten einer Vermarktlichung der Gesellschaft wird Margaret Thatcher zugeschrieben.
} 
die Diskussion über die technologische Revolution und die tatsächliche Nutzung von Technologien den Umbruch der Arbeitsformen und Arbeitsbeziehungen im Sinne der neoliberalen Agenda mit vorantreiben. Dafür wird am Beispiel der sogenannten Plattformwirtschaft gezeigt, dass Umwälzungen in den Arbeitsbeziehungen die neue soziale Frage der Prekarisierung verschärfen und eher in dieser Hinsicht als im Hinblick auf technologische Umbrüche ein Vergleich mit der ersten Industriellen Revolution angemessen erscheint.

\section{Beschäftigungswirkungen von Industrie 4.0 und Digitalisierung}

In den Diskussionen über die Digitalisierung wird das Revolutionäre der Technik sehr oft in der Möglichkeit gesehen, menschliche Arbeit zu ersetzen. Von industrieller Automatisierung und selbstfahrendem Automobil über Übersetzungsprogramme und Chatbots, welche Kundenanfragen automatisch beantworten, bis zum Roboter im Pflegeheim reichen die Beispiele. Dabei werden die Auswirkungen der Digitalisierung auf die Beschäftigung, also die Zahl der Arbeitsplätze, sehr unterschiedlich beurteilt. Die Medien kolportieren häufig Schreckensszenarien hoher Ersetzbarkeit von Arbeitsplätzen und daraus folgender Arbeitslosigkeit. Eine Grundlage für diese Voraussagen war die vielzitierte Oxford-Studie (Frey/Osborne 2013). Die Autoren beschrieben das Automationsrisiko von Berufen anhand ihres Gehalts an Routinetätigkeiten, aber auch an Nicht-Routinetätigkeiten, sofern sie durch die Weiterentwicklung der Technologie in wohldefinierte Probleme umgewandelt werden können. Darüber hinaus berücksichtigten sie Hindernisse für die Automatisierung in Form des Bedarfs an sozialer Intelligenz, Kreativität oder Wahrnehmungsfähigkeiten. Sie schätzten auf dieser Basis, dass 47\% aller Arbeitsplätze in den USA in den nächsten Jahren automatisierbar seien.

Andere Studien gingen in eine ähnliche Richtung oder gaben Entwarnung. Ein Beispiel für Letzteres ist die Untersuchung des Instituts für Arbeitsmarkt- und Berufsforschung (IAB) über die Beschäftigungswirkungen von Industrie 4.0 in Deutschland (Wolter et al. 2015). Darin wurden bis 2020250.000 zusätzliche Arbeitsplätze und der Wegfall von 260.000 Arbeitsplätzen erwartet. 2030 sollen 460.000 wegfallenden Arbeitsplätzen 400.000 zusätzliche gegenüberstehen. Das bedeutet, dass die Beschäftigungswirkungen im Saldo relativ gering sind, dass es aber einige Verschiebungen in einer Vielzahl an Berufsfeldern geben wird. Bestimmte Tätigkeiten und Berufe werden an Bedeutung verlieren, entsprechende Verluste von Arbeitsplätzen sind zu erwarten. Dem stehen aber neue Jobs gegenüber. Damit wiederholt sich ein alter Befund der Arbeits- und Industriesoziologie, nämlich, dass es Rationalisierungsverlierer/innen und Rationalisierungsgewinner/innen gibt (Kern/Schumann 1984), dass Tätigkeiten wegfallen und andere dazu kommen, Berufe an Bedeutung verlieren, andere an Bedeutung gewinnen oder überhaupt neu entstehen.

Arntz und Kollegen (2016) bezweifeln ebenfalls die Befunde von Frey und Osborne (2013). Letztere hätten durch ihre Konzentration auf automatisierbare Berufe den Beschäftigungsverlust drastisch überschätzt. Denn nicht alle Tätigkeiten eines Berufs seien gleichermassen automatisierbar. So kommen bei Buchhalterinnen oder bei Verkäufern im Einzelhandel, die laut Frey und Osborne mit einem Automationspotenzial von 98 Prozent bzw. 92 Prozent konfrontiert sind, auch Gruppenarbeiten und persönliche Gespräche vor, die nicht automatisierbar seien. Berücksichtige man nun die Vielfalt und Unterschiedlichkeit von Tätig- 
keiten innerhalb der Berufe, ergebe sich, dass im Durchschnitt der 21 untersuchten OECDLänder 9 Prozent der Arbeitsplätze automatisierbar seien. Für Deutschland, wie auch für Österreich, wird dieser Wert auf 12 Prozent geschätzt. ${ }^{3}$ Die OECD geht davon aus, dass die Schweiz wegen ihrer Berufsstruktur stärker als Deutschland von negativen Folgen der Digitalisierung auf die Beschäftigung betroffen sein könnte (OECD 2017: 121).

Für Österreich ergab eine Schätzung von Nagl und Kolleginnen (2017), dass insgesamt 9 Prozent der Beschäftigten von einem hohen Automatisierungsrisiko betroffen sind. Mit Letzterem ist gemeint, dass ihr Arbeitsplatz mit einer Wahrscheinlichkeit von über 70 Prozent automatisiert wird. Auch dies ist zwar ein deutlich niedrigerer Wert als der von Frey und Osborne genannte. Dennoch stellt sich die Frage, ob er angesichts der schon bestehenden hohen Arbeitslosigkeit durch Beschäftigungspolitik bewältigt werden kann. Zudem ist zu bedenken, dass die Tätigkeiten nicht gleichbleiben. So gibt es inzwischen genügend Erfahrung damit, dass Kundinnen und Kunden etwa bei der Einführung der Callcenter oder bei Automation eine Verschlechterung des Service schlicht akzeptieren müssen, wenn sie nicht zur auserlesenen Gruppe der besonders Zahlungskräftigen gehören. Insofern ist das persönliche Gespräch im Handel oder Bankwesen alles andere als eine Konstante.

Damit trägt eine auf konkrete Berufe und Arbeitsplätze bezogene Betrachtungsweise zwar zur besseren Einschätzung möglicher Wirkungen der Digitalisierung bei. Wichtige Verschiebungen, die gerne dem disruptiven Charakter der Technologie zugeschrieben werden, bleiben aber unberücksichtigt. Doch schon seit den 1980er Jahren besteht ein wichtiger Teil der arbeitssparenden Veränderungen in einer "systemischen Rationalisierung“ (Altmann et al. 1986), das heißt, es werden Teilprozesse informationstechnisch verbunden und zunehmend betriebsübergreifende und zwischenbetriebliche Wertschöpfungsketten zum Gegenstand der Rationalisierung. Damit wird nicht nur Arbeit am einzelnen Arbeitsplatz effizienter oder automatisiert, sondern es können Verarbeitungsstufen überhaupt obsolet werden. Unter dem Stichwort business-to-business beispielsweise werden diese Strategien schon längere Zeit verfolgt.

Dies betrifft zudem in einer räumlichen Dimension die Möglichkeiten der geografischen Verlagerung von Arbeit, die sich in einem Verlust an Arbeitsplätzen in einer Region und einem Zuwachs in einer anderen niederschlagen. Informatisierung und elektronische Vernetzung sind für das, was Harvey (2001) als „spatial fix“ bezeichnet hat, höchst relevant: Zum einen können entfernte Standorte leichter in den Produktions- und Dienstleistungsprozess einbezogen und Arbeit dorthin verlegt werden, weil die Koordination erheblich erleichtert wird. Zum anderen wird die Digitalisierung genutzt, um die Bindung von Arbeitsprozessen an einen Ort zu schwächen, Arbeit also ortsunabhängiger zu machen (Flecker/Schönauer 2016), womit sich der Zeitraum der räumlichen Fixiertheit des Kapitals verkürzt. Bereits in den letzten beiden Jahrzehnten ermöglichte die Digitalisierung der Arbeit einen erheblichen Wandel in der internationalen Arbeitsteilung. Die Verlagerung von Arbeit aus Nordamerika und Westeuropa nach Asien hat in Ländern wie Indien, Vietnam und China zu einer großen Zahl an Arbeitsplätzen im Bereich der Software-Entwicklung, der Produktentwicklung, des Business Process Outsourcing oder der IT enabled services geführt. Auch wenn digitale Arbeit nicht

\footnotetext{
${ }^{3}$ Für die Schweiz konnte in dieser Studie kein Vergleichswert ermittelt werden, weil das Land nicht an der PIAACStudie teilnahm.
} 
per se ortsunabhängig ist, haben technische Entwicklung und Reorganisation der Unternehmen und Produktionsnetzwerke wie erwähnt dazu beigetragen, dass Unternehmen im Hinblick auf den Standort für digitale Arbeit große Wahlmöglichkeiten haben. Dabei sind drei Formen der Verlagerung von Arbeit zu unterscheiden: Erstens werden Konzerne ständig organisatorisch und räumlich umstrukturiert. Von IBM heißt es, dass das Unternehmen seit 2012 mehr Personen in Indien beschäftigt als in den USA (Goel 2017). Zweitens kann die Auslagerung von Arbeit aus Unternehmen und öffentlichen Organisationen an Dienstleistungsunternehmen vor Ort, im selben Land oder aber grenzüberschreitend erfolgen. Damit haben Auslagerungen und Umstrukturierungen von Wertschöpfungsketten Auswirkungen auf regionale Arbeitsmärkte. Schließlich erfolgt die Aus- und Verlagerung von Arbeit zunehmend über Internetplattformen für Crowdsourcing oder Crowdworking (siehe dazu unten). Zwar wird die Verbreitung dieser Form der Arbeitsvermittlung bisher noch als marginal eingestuft (Mandl et al. 2015: 111), doch könnte sie größere Bedeutung erlangen, wenn sich Unternehmen dieser Vermittlungsdienste in stärkerem Ausmaß bedienen.

Von Analysen, die auf Arbeitsplätze fokussieren, wird die Frage der Einbeziehung der Kundinnen/Kunden und der Bürgerinnen/Bürger in die Wertschöpfung bzw. Verwaltung ebenso wenig erfasst. Im Zuge der Digitalisierung wurde die Selbstbedienung auf neue Bereiche ausgeweitet, aber auch in den klassischen Bereichen, wie dem Einzelhandel oder im Bankwesen, weiter forciert. Gerade im Zusammenhang mit der zunehmenden Abwicklung von Geschäften über das Internet wird Druck auf die Konsumentinnen/Konsumenten ausgeübt, einen immer größeren Teil der Arbeit zu übernehmen. Oft findet man auf den Internetseiten keine Kontaktdaten von Mitarbeiter/innen, kann diese über Telefon nur sehr schwer erreichen und ist daher gezwungen, die Arbeit zu erledigen, die mit der Produktauswahl, der Dateneingabe, der Bezahlung etc. verbunden ist (Ritzer et al. 2012: 385). Entsprechend groß können die Beschäftigungswirkungen der Übertragung von Arbeitsaufgaben auf die Konsument/innen eingeschätzt werden.

Die Bereiche von bezahlter und unbezahlter Arbeit verschwimmen aber auf weitere Arten. So wird die Produktion von Inhalten für das Internet zu einem großen Teil in Form unbezahlter Arbeit geleistet. Dies betrifft nicht nur die Digital Commons, wie Wikipedia, sondern auch gewinnorientierte Internetunternehmen. Während die Aktivität von Bloggerinnen und Bloggern zunächst als Hobby oder als zusätzliche Möglichkeit der Selbstdarstellung neben der Erwerbstätigkeit erscheint, stellt die Verschiebung von professioneller Medienarbeit in unbezahlte Arbeit tatsächlich eine Bedrohung der Erwerbschancen von Journalistinnen und Journalisten dar. Waren schon die Leserbriefe an Zeitungen unbezahlte Arbeit, so nehmen die Kommentare und anderen Beiträge der Internetnutzerinnen und -nutzer in den Onlinemedien einen immer größeren Raum ein. Mehr noch: Diejenigen, die in diesem Feld berufstätig sein wollen und nicht zu den Etablierten und gut Bezahlten gehören, werden - so Einschätzungen aus den USA - vor die Alternative gestellt, entweder unterbezahlt unkreative Arbeit zu leisten oder sich ohne Bezahlung als BloggerIn zu verwirklichen:

After all, the alternative to churning out junk products for a content farm is to play the reputational game by posting for free, like the Huffington Post bloggers. The former option involves the kind of routine toil that is anathema to aspiring creative professionals. The 
latter option promises the kind of unalienated expression of thought that is closer to their ideal. Yet only one of those will guarantee food on the table. (Ross 2013: 15)

Eine noch weniger sichtbare Form der Verwandlung bezahlter Arbeit in unbezahlte wird als Gamification bezeichnet. Dabei wird Arbeit als Spiel gestaltet, bei dem es beispielweise um das Erkennen von Buchstaben oder Wörtern geht, um so die Kosten für die Dateneingabe zu reduzieren. Auch die Sicherheitsprüfungen von reCaptcha stellen unbezahlte Arbeit dar: Wenn wir bei Anfragen im Internet zeigen müssen, dass wir keine Roboter sind, kategorisieren wir z.B. Bilder. Die entsprechende bezahlte Arbeit wird dadurch ersetzt. Alle diese vielfältigen Entwicklungen müssen berücksichtigt werden, will man die Veränderung der Beschäftigung im Zuge der Digitalisierung abschätzen. Die Gründe für die zu beachtenden Dynamiken erweisen sich als sehr vielfältig. Sie hängen häufig mit den Möglichkeiten zusammen, die durch digitale Technologien entstehen, sind jedoch nie allein diesen zuzuschreiben. Tendenzen des ökonomischen Strukturwandels, wie jener der Ausbreitung des Online-Handels auf Kosten des stationären Einzelhandels ausgelöste, können nicht nur einen Verlust an Arbeitsplätzen, sondern auch eine Verschiebung von qualifizierter Arbeit zu unqualifizierter beinhalten. Die Macht der Konsumenten und Konsumentinnen spielt keine geringe Rolle, wenn es um die Umwandlung von bezahlter in unbezahlte Arbeit geht. Schließlich ist schwer abschätzbar, in welchen Formen die Anerkennungsbedürfnisse und die Geltungssucht der Menschen künftig ausgelebt werden, die das Geschehen in den sozialen Medien stark prägen.

Letztlich entscheidet die Verteilung des gesellschaftlichen Reichtums darüber, ob es zu einer großen Nachfrage für neue, technisch ermöglichte Produkte und Dienstleistungen kommt, welche die beschriebenen negativen Beschäftigungswirkungen kompensieren könnten. Dabei geht es nicht nur um die Einkommensverteilung im Sinne der Höhe von Löhnen und Gehältern, sondern auch um die Höhe der staatlichen Transferleistungen, die sich als höchst wirksam dafür erwiesen haben, Ungleichheit zu mildern und Armut zu begrenzen. Das heißt aber zugleich, dass sie die kaufkräftige Nachfrage stützen, insbesondere angesichts der notwendigerweise hohen Konsumneigung der Empfänger/innen von sozialen Transferzahlungen. Ist es angesichts der hier angedeuteten Komplexität der Zusammenhänge zwischen technischem Wandel und Beschäftigung schon schwer, belastbare Aussagen über die Folgen der Digitalisierung auf die Anzahl der Arbeitsplätze zu gewinnen, so kann auch nicht schlicht vorausgesetzt werden, worin denn die technologischen Innovationen bestehen und wie man sie wohl weiter vorantreiben wird. Welche Form die Technik annimmt und für welche Anwendungen sie genutzt wird, ist aber nicht unerheblich für die Fragen nach den quantitativen und qualitativen Auswirkungen auf die Erwerbsarbeit.

\section{Technikdeterminismus oder Social Shaping?}

Unter dem Schlagwort Industrie 4.0 wird seit einigen Jahren im deutschsprachigen Raum die Debatte über einen bevorstehenden Technisierungsschub in der industriellen Produktion geführt. Die Bezeichnung spielt auf die Behauptung einer vierten industriellen Revolution an. Inhaltlich geht es um den Einsatz sogenannter cyberphysikalischer Systeme, bei denen Maschinen, Behälter, Materialien und Produkte mit Chips ausgestattet sind, die für die direkte Kom- 
munikation zwischen diesen Artefakten genutzt werden. Damit sollen umfassende Produktionsprozesse ohne menschlichen Eingriff auskommen. „In einer Vision der flächendeckenden Durchdringung dieses Ansatzes steuern sich die Aufträge selbstständig durch ganze Wertschöpfungsketten, buchen ihre Bearbeitungsmaschinen und ihr Material und organisieren ihre Auslieferung zum Kunden" (Spath 2013: 22).

Wie auch frühere vergleichbare Debatten sind die aktuellen Diskussionen über Industrie 4.0 und Digitalisierung noch immer von der Vorstellung geprägt, „dass technischer Fortschritt gemäß der Natur der Dinge abrollen muss; (...) dass die Gesellschaft sich dem technischen Fortschritt anzupassen hat (...) (und) dass das Potential technischen Fortschritts nur ausgeschöpft werden kann, wenn ganz bestimmte Qualifikationsstrukturen“ erreicht werden, wie Burkhard Lutz (1979: 8) vor bald 40 Jahren schrieb ${ }^{4}$. Er warnte vor dieser Perspektive, welche die sozialen Grundlagen und die Gestaltungsmöglichkeiten sowohl der Technikentwicklung als auch des Technikeinsatzes und der Arbeitsorganisation negiert. Schon lange hat sich in den Sozialwissenschaften demgegenüber die Überzeugung durchgesetzt, dass der Wandel der Arbeit nicht von technischen Innovationen bestimmt und die Entwicklung der Technik nicht ihrer naturwissenschaftlich basierten Eigenlogik folge. Vielmehr bestimmen entsprechend der theoretischen Perspektive des "Social Shaping of Technology" (MacKenzie/Wajcman 1985; Williams/Edge 1996) umgekehrt gesellschaftliche Akteure und Prozesse die Entwicklung der Technik und sind auch für die Folgen des Technikeinsatzes auf die Arbeit verantwortlich. Es wird dabei nicht geleugnet, dass technische Innovationen meist soziale Wirkungen haben. Vielmehr wird gezeigt, dass Erfindungen und technische Lösungen für gewisse praktische Probleme sich nicht aufgrund ihrer technischen Überlegenheit allein durchsetzen. Der Prozess der Entwicklung und der Anwendung neuer Techniken umfasst eine große Zahl von Entscheidungen zwischen technischen Optionen. Und welche Option jeweils gewählt wird, hängt von einer Reihe von sozialen Faktoren ab, welche daher die Gestaltung der Technik und damit auch ihre sozialen Auswirkungen formen (Wajcman 2015: 28).

Wie beispielsweise Informations- und Kommunikationstechnologien aufgebaut sind, lässt sich auf soziale Beziehungen und politische Entscheidungen zurückführen. Die für uns heute selbstverständliche Trennung von Software und Hardware und die Möglichkeit, verschiedenste Programme für unsere Computer zu kaufen, geht auf eine politische Intervention zur Beschränkung der Marktmacht des Unternehmens IBM zurück. IBM durfte Hard- und Software nicht mehr als eine Einheit verkaufen, was die Entstehung eines Softwaremarktes ermöglichte (Williams/Edge 1996: 880). In einer klassischen Studie über die soziale Formung der Technik analysierte David Noble (1979) die Entscheidungen, die bei der Entwicklung der programmierbaren Werkzeugmaschinen für die Industrie getroffen wurden. Bei den NC-Maschinen (numerical control) wurde die bisherige Produktionsarbeit der Facharbeiter/innen in die des Programmierers und die der Maschinenbedienerin aufgespalten, wobei die Maschinenbedienerin keine Facharbeiterin sein musste, weil sie im Wesentlichen die Teile einspannte und nach der automatischen Bearbeitung wieder entnahm. Das folgte aus dem zentralen Ziel der Entwicklung von NC-Maschinen, nämlich die Produktion von den Qualifikationen der gelern-

\footnotetext{
${ }^{4}$ Auf das Zitat wurde ich durch Pfeiffer (2010: 240) aufmerksam.
} 
ten Dreher/innen und Fräser/innen unabhängig zu machen. Mit der Ausweitung der Anwendung der NC-Technik außerhalb der Rüstungsindustrie wurden die sozialen Einflüsse vielfältiger. Es kam zu Aushandlungen über die Technik zwischen Unternehmensleitungen, verschiedenen Gruppen von Arbeitenden und ihren Gewerkschaften. Eine flexiblere Variante der Maschinensteuerung, genannt computer numerical control (CNC), setzte sich durch, bei der man Computer zur Programmierung direkt an den Maschinen anbrachte. Das ermöglichte die Programmierung in der Werkstatt durch die Arbeiter/innen selbst und ließ deshalb unterschiedliche Formen der Arbeitsteilung zu. Während in Deutschland aufgrund der großen Bedeutung der Facharbeit häufiger Werkstattprogrammierung anzutreffen war, wurde in Großbritannien eine stärkere Arbeitsteilung zwischen Programmierung, Maschinenbedienung und -beaufsichtigung üblich (Williams/Edge 1996: 885).

Wenn über die Auswirkungen der Digitalisierung auf die Erwerbsarbeit gesprochen wird, kann man also nicht davon ausgehen, dass eine eigenlogisch voranschreitende Entwicklung der Informationstechnik die Ursache von Veränderungen in der Arbeit wäre. Vielmehr ist schon die Technologieentwicklung, also der technische Innovationsprozess, als gesellschaftlicher Prozess zu sehen. Auch in der Anwendung der Technik spielen verschiedenste soziale Interessen und Einflüsse eine Rolle. Genau genommen wird die Informations- und Kommunikationstechnik erst im Prozess der Anwendung geformt. So geht es beim Internet nicht nur um die Vernetzung von Computern und die Informationsübertragung auf Basis des entsprechenden Protokolls. Die derzeit wieder aufgeflammte Diskussion über die Netzneutralität, also die Gleichbehandlung der verschiedensten Datenpakete, die im Internet befördert werden, zeigt auf, wo unterschiedliche gesellschaftliche Interessen ansetzen. Während die eine Seite die Gleichbehandlung gesichert wissen will, verlangt die andere, dass die Datenpakete jener Firmen oder Nutzer schneller vorankommen sollen, die dafür bezahlen. Technisch sind freilich beide Optionen umsetzbar. Auch das Verhalten der Millionen Nutzer/innen bestimmt die Gestalt des Internet tagtäglich mit, wenn es beispielweise darum geht, welche Websites stark und welche schwach verlinkt sind, was sich auf die Suchergebnisse mittels Google, Ecosia oder Yahoo auswirkt (Wajcman 2015: 30). Das trifft aber auch auf unseren PC oder unser Smartphone zu, von deren vielfältigen Funktionen wir nur einen Bruchteil tatsächlich nutzen, sodass wir die grundsätzlich verfügbare Technik eingeschränkend formen. Insgesamt verursacht die Technik letztlich nicht die Veränderungen in der Arbeit, sie erleichtert oder ermöglicht vielmehr bestimmte Geschäftsmodelle, Organisationsformen und Rationalisierungsschritte (Pfeiffer 2012). Dies ist sehr deutlich an der sogenannten Plattformwirtschaft zu sehen, in der Internetunternehmen Aufträge zwischen Kund/innen und Arbeitenden vermitteln und dabei eine ganz spezifische Gestaltung der Kommunikationsbeziehungen, der Geschäftsprozesse und der Überwachung durch die Auswertung von Prozessdaten vornehmen. Auf dieses Beispiel werde ich weiter unten zurückkommen.

\section{Die Ausweitung der Optionen}

Die Digitalisierung ermöglicht also weitgehende Veränderungen und erweitert damit den Optionsspielraum für die Anwendung von Technik und die Gestaltung von Organisation und Arbeit erheblich. Von disruptiver Technologie ist die Rede, wenn diese Chancen dafür genutzt 
werden, bisherige Produkte, Markt- und Organisationsformen oder Geschäftsbeziehungen durch neue Angebote auszuhebeln. Der Online-Handel durch Amazon und viele andere Unternehmen, der Ersatz für Hotelzimmer durch AirBnB oder der Taxidienst Uber sind die bekanntesten Beispiele dafür. Der damit bewirkte Strukturwandel bedeutet immer auch eine Veränderung der Arbeitsformen und der Besteuerung. Der Optionsspielraum erweitert sich auch im Hinblick darauf, was Inhalt einzelner Berufe ist und welche Tätigkeiten zu Berufen oder an einzelnen Arbeitsplätzen gebündelt werden. So sind beispielsweise nur noch wenige Arbeitsplätze vorhanden, an denen Texte nach Diktat getippt werden, weil seit der Verbreitung von Personal Computern die Eingabe von Text in das Tätigkeitsbündel jener integriert ist, die früher Schriftstücke diktierten. Sogar Präsidenten scheinen heute ihre Tweets selbst zu schreiben. Die erweiterte Optionsvielfalt zeigt sich auch daran, dass die organisatorisch bestimmten Arbeitsabläufe und Kooperationsformen in der Gestaltung der Informations- und Kommunikationstechnik festgelegt werden können. Ist die Bearbeitung von Rechnungen, Bestellungen, Steuererklärungen etc. vollständig digitalisiert, kommt sie also ohne Information auf Papier aus, so lässt sich über den elektronischen Workflow bestimmen, wie die Bearbeitungsschritte an den einzelnen Arbeitsplätzen ineinanderzugreifen haben.

So gesehen ist es eigentlich verfehlt, nach den Folgen der Digitalisierung auf die Arbeit zu fragen. Vielmehr erweitert die derzeitige (soziale und politische) Gestaltung der IKT die Optionen für die primär betriebswirtschaftlich ausgerichteten und in gesellschaftspolitische Auseinandersetzungen eingebetteten Entscheidungen über die Arbeitsorganisation. Erst daraus ergeben sich die als Aufwertung oder Degradierung von Arbeit erkennbaren Folgen sowie die Entwicklung, welche die Steuerung und Kontrolle der Arbeit nimmt. Auch in räumlicher Hinsicht kam es zu einer entscheidenden Erweiterung der Optionen für die Gestaltung von Arbeit. Wenn die ortsgebundenen von den ortsunabhängigen Tätigkeiten getrennt werden und zu erledigende Aufgaben durch Digitalisierung der Information ortsunabhängiger werden, sind neue Voraussetzungen für die geografische Verlagerung von Arbeit bis hin zu neuen Formen der internationalen Arbeitsteilung geschaffen (Flecker/Schönauer 2016). Damit wird eine neue Dimension in der Mobilität des Kapitals erreicht: In den 1970er und 80er Jahren war vom Übergang von multinationalen zu transnationalen Konzernen die Rede gewesen. Der Unterschied lag darin, dass in der Phase der Internationalisierung mit multinationaler Unternehmensform alle Unternehmensfunktionen von der Produktentwicklung über die Produktion bis zu Verwaltung und Marketing im jeweiligen Land angesiedelt wurden, die Tochtergesellschaften also eine Nachbildung der Muttergesellschaft waren. Transnationale Unternehmen hingegen bilden ein grenzüberschreitend geografisch verteiltes Netz an Forschungslabors, Produktionsstätten, Rechenzentren, Verwaltungseinheiten etc. Daher können solche Konzerne für jede dieser Unternehmensfunktionen getrennt die jeweils am günstigsten erscheinenden Länder und Städte wählen, wodurch sie insgesamt erheblich mobiler werden (Morgan 2005). Diese Mobilität stellt umgekehrt ein Drohpotenzial gegenüber Staaten, Regionen und Städten sowie gegenüber den Arbeitenden dar. Auf Basis der Digitalisierung und unter Nutzung elektronischer Netzwerke wird diese Mobilität weiter erhöht und deutlich feingliedriger: Welchem Standort im Konzern ein Auftrag etwa für die Entwicklung einer Software erteilt wird, wo Teilaufgaben dieses Softwareprojekts ausgeführt, wo Verwaltungsarbeiten erledigt, von wo aus Kunden über Callcenter betreut werden - diese und viele andere 
Fragen können in kürzeren Abständen neu beantwortet werden. Mehr noch: Projektteams etwa in der Produktentwicklung werden geografisch verteilt zusammengesetzt (Will-Zocholl 2016) oder Anrufe von Kundinnen und Kunden werden je nach Auslastung von Callcentern automatisch über große Distanzen und Landesgrenzen weitergeleitet. Nach der Verlagerung von Produktion in Länder mit niedrigen Löhnen und wenigen Umweltauflagen ist seit den 2000er Jahren auf Basis der Digitalisierung zunehmend eine globale Verteilung der Kopfarbeit (Boes/Kämpf 2011) zu beobachten.

Nicht nur für die Gestaltung der Organisation und der Arbeitsabläufe innerhalb von Unternehmen hat die Digitalisierung zusätzliche Optionen gebracht. Informations- und Kommunikationstechnologien bilden auch das Rückgrat für die Auslagerung von Aufgaben aus dem Unternehmen und für die immer komplexeren Zulieferbeziehungen. Erst die zusätzlichen Möglichkeiten der Steuerung und Kontrolle, welche diese Technologien bieten, haben die heute vielfach aufgespaltenen Unternehmensstrukturen und die weitverzweigten Firmennetzwerke zu wirtschaftlich sinnvollen Optionen gemacht (Weil 2014). Damit sind erhebliche Auswirkungen auf die Beschäftigungs- und Arbeitsbedingungen sowie die Chancen von Behörden verbunden, Standards des Arbeitsschutzes durchzusetzen. Ein anschauliches Beispiel dafür ist die Paketzustellung, bei der Streifencodes, Chips und GPS die Steuerung des Versands und die Verfolgung von Sendungen ermöglichen. Während die großen, global agierenden Unternehmen wie DHL, TNT oder UPS die Logistik und den Transport im Detail und auf die Minute steuern, sind zwischen ihnen und den Paketzustellern vor Ort mehrere Stufen von Subauftragnehmern zwischengeschaltet. Zwar garantieren die großen Paketdienstleister den Kundinnen und Kunden kurzfristige und verlässliche Zustellung und überwachen daher die Lieferung elektronisch, doch sind sie für die Beschäftigung der Zusteller/innen und damit für die Arbeits- und Beschäftigungsbedingungen nicht zuständig. Ihre Vorgaben und der große Konkurrenzdruck in dem leicht zugänglichen Markt führen jedoch zu prekärer, häufig scheinselbstständiger Beschäftigung und niedrigen Einkommen (Haidinger 2012). Die Digitalisierung hat, wie dieses Beispiel anschaulich zeigt, die Einführung einer Organisationsform erleichtert, in der Firmen zwar eine umfassende und detaillierte Kontrolle über die Produktion oder Dienstleistung ausüben können, aber keine Verantwortung für die Arbeitsbedingungen übernehmen (Weil 2014). Ist der Marktzugang für Unternehmer/innen am unteren Ende der Wertschöpfungsketten einfach, wie etwa in der Paketzustellung oder auch in der Bekleidungsherstellung, so herrscht große Konkurrenz, die auf Kosten der Löhne und Arbeitsbedingungen ausgetragen wird. Vielfach kommt es zu einer Informalisierung der Arbeit und Hinterziehung von Abgaben, die gerade dadurch von den Behörden schwer zu verhindern ist, dass Betriebe leicht zu eröffnen sind, aber auch häufig wieder geschlossen werden.

Wie diese Ausführungen anschaulich machen sollen, besteht eine angeblich technologische Revolution vor allem darin, dass die auf Technik als Ermöglicherin zurückgehende Optionsvielfalt in einer bestimmten Art und Weise genutzt wird. Die Automation von Arbeitsschritten, die vielfach bei der Analyse möglicher Beschäftigungswirkungen der Digitalisierung im Vordergrund steht, ist nur ein Aspekt - und nicht der wichtigste. Vielmehr hat insbesondere die Art und Weise, wie die erweiterten Möglichkeiten der Digitalisierung abseits der Automation einzelner Arbeitsplätze genutzt werden, große Auswirkungen auf die Zukunft der Arbeit: Bleibt das „internalisierte Beschäftigungsverhältnis“ (Rubery 2010), also die Anstellung im 
Unternehmen, und insbesondere das Normalarbeitsverhältnis erhalten oder wird die Auslagerung in Selbstständigkeit verstärkt? Werden Aufgaben noch weiter in Subunternehmen ausgelagert, die Wertschöpfungsketten dadurch verlängert, an deren unteren Ende informelle Arbeit, hohe Flexibilitätsanforderungen und oft gesundheitsgefährdende Arbeitsbedingungen zu finden sind (Frade/Darmon 2005)?

Aber auch bei Fragen der Automation sind gesellschaftliche Interessen und politische Zielsetzungen relevant. Was automatisiert wird, hängt weniger von den technischen Möglichkeiten als vielmehr von betriebswirtschaftlichen Kalkulationen und von den „Produktionskonzepten“ (Kern/Schumann 1984) ab, die entweder das Ziel verfolgen, die Produktion möglichst unabhängig von Arbeiter/innen werden zu lassen, oder aber die Beiträge der menschlichen Arbeit wertschätzen und entsprechende Spielräume dafür schaffen. Möglichst weitgehende Automation und Beschränkung des Einflusses der lebendigen Arbeit ist ein ebenso betriebswirtschaftliches wie politisches Ziel, während sich die Nutzung der Fähigkeiten und Erfahrungen der Arbeitenden im Sinne eines „subjektivierend-erfahrungsgeleiteten Arbeitens“ (Böhle 2010) betriebswirtschaftlich ebenso, wenn nicht noch besser rechnen kann. Je nach Produktionskonzept kann auch bei Industrie 4.0 zwischen einem Automatisierungsszenario, das einen möglichst sich selbst steuernden, technischen Produktionsprozess zum Ziel hat, einerseits und einem Werkzeugszenario unterschieden werden, in dem die Technik die Arbeitenden unterstützt (Windelband/Spöttl 2012). Um ein Beispiel zu geben: In der Branche Transport und Logistik werden die Lkw-Transporte mittels komplexer Informationssysteme gesteuert, und die Lkw sind mit Bordcomputern ausgestattet, die mit der Zentrale vernetzt sind. Verwenden Lkw-Fahrer/innen diese Tourenmanagementsysteme ihres Bordcomputers als Hilfsmittel und können sie die Reihenfolge der Aufträge selbst bestimmen, neue Aufträge hinzunehmen und direkt mit Kundinnen und Kunden kommunizieren, so entspricht diese Gestaltung einem Werkzeugszenario. Werden aber die Touren auf Basis automatisiert erfasster Daten vollständig vom System geplant und dem Fahrer bzw. der Fahrerin vorgegeben, büßt dieser/diese Handlungsspielräume und Kompetenzen ein (Windelband et al. 2010). Es kann den Fahrer/innen auf Grundlage der laufend erfassten Fahrzeugdaten sogar angeraten werden, in einen höheren Gang zu schalten, um Treibstoff zu sparen.

\section{Die Qualität der Arbeit}

Die Auslegung der Technik in Verbindung mit der Gestaltung der Arbeitsorganisation hat tiefgreifende Auswirkungen auf die Qualität der Arbeit. Das bedeutet auch, dass die Folgen nicht auf eine bestimmte Technik im Allgemeinen zurückgeführt werden können. Dagegen wurden in der Diskussion über die 4. Industrielle Revolution dem vertretenen Technikdeterminismus entsprechend generelle Folgen für die Arbeit angenommen. Die dabei verwendeten Slogans ähnelten jenen der früheren Automatisierungsdebatten: Der Mensch wird frei für kreative Aufgaben. Entgegen diesen und ähnlichen Erwartungen, die schon die Diskussion über die Wissensgesellschaft vor etwa zwei Jahrzehnten prägten, zeigen Befunde der Arbeitsforschung, dass die Digitalisierung von Arbeit höchst unterschiedliche Wirkungen zeitigt. Während zum Teil tatsächlich eine Ausweitung von Handlungsspielräumen, Höherqualifizierung und partizipative Managementformen zu beobachten sind, finden sich im Gegensatz dazu auf 
fortgeschrittenem Technologieniveau auch hoch arbeitsteilige, standardisierte und dequalifizierende Formen der Arbeitsgestaltung.

Für höhere Qualifikationsanforderungen an einen Teil der Beschäftigten spricht, dass ihnen in bestimmten, wenn auch nicht in allen Produktionskonzepten, die Rolle der Entscheider/innen und Steuer/innen zugesprochen wird. Problemlösungsfähigkeiten, selbstgesteuertes Handeln, Kommunikationsfähigkeiten werden neben den technischen Sach- und Anwendungskenntnissen gefordert, insbesondere dann, wenn die Facharbeit durch Planungsund Entscheidungsaufgaben angereichert wird (Holtgrewe et al. 2016). Dazu müssen die Arbeitenden breit qualifiziert sein und durch eine lernförderliche Arbeitsorganisation sowie eine breit angelegte Weiterbildung unterstützt werden, die selbstständiges Arbeiten fördert und als aktives Instrument systematischer Personalentwicklung und Aufstiegsförderung konzipiert ist (Promotorengruppe Kommunikation der Forschungsunion Wirtschaft - Wissenschaft 2013: 57).

Am anderen Ende der Polarität von Arbeitsorganisationsformen und Qualifikationsanforderungen steht die Degradierung von Arbeit, für welche sich ebenfalls viele Beispiele finden lassen - von der Sachbearbeitung in "Shared Service Centers" (Howcroft/Richardson 2012) oder im öffentlichen Dienst (Carter et al. 2011) bis zur Kundinnen-/Kundenbetreuung über Call- oder Contactcenter (Bain et al. 2002; Sproll 2016). Dabei wird Arbeit aus ihren bisherigen Kontexten gelöst, in einzelne Verrichtungen aufgegliedert, für welche standardisierte Vorgaben entwickelt werden. Ziel und Folge dieser Rationalisierungsstrategie ist die Ersetzbarkeit und Verbilligung der Arbeitskraft sowie ihre gesteigerte Kontrollierbarkeit. Bei digitaler Arbeit kommt hinzu, dass die Standardisierung die Auslagerung und die geografische Verlagerung der Arbeit erleichtert - wie auch die Maßnahmen im Zuge von Aus- und Verlagerungen häufig die Standardisierung vorantreiben (Flecker/Meil 2010; Ramioul/van Houtegem 2015).

In degradierter ebenso wie in hochqualifizierter Arbeit ermöglichte die Digitalisierung eine Beschleunigung, die sich in der industriellen Produktion und der Kommunikation am deutlichsten erkennen lässt. Paradoxerweise führte die Beschleunigung aber nicht zu einem Mehr an freier Zeit und zu einem entspannteren Arbeitsrhythmus. Im Gegenteil wird die Beschleunigung des Lebenstempos vielfach auf die Technologie zurückgeführt (Rosa 2005). Ein Aspekt davon ist die Entgrenzung der Arbeit. Die Verwendung von Laptops und Smartphones führte dazu, dass die Arbeit nicht notwendigerweise im Büro zurückbleibt, wenn man dieses verlässt. So beschreibt Melissa Gregg (2011) in ihrer Untersuchung Work's Intimacy über Informations-, Kommunikations- und Bildungsberufe, dass es gerade die bequeme Verwendbarkeit der Geräte ist, die dazu verlockt, neben anderen Tätigkeiten zu Hause ständig mit der Erwerbsarbeit verbunden zu bleiben. Die ohnehin schon bestehende starke psychische Verbindung zu dieser Arbeit wird also noch verstärkt. Dabei ist aber häufig unklar, was zur Erwerbsarbeit zählt und was nicht. Aus Angst vor überbordenden Eingangsordnern im EMail-Programm, um Dinge weiterzutreiben oder um angesichts der vielen Arbeit und der Millionen Deadlines einfach nur bei Verstand zu bleiben, opfern die Befragten gerne einige Zeit zu Hause für das Abrufen von E-Mails und für kleine Erledigungen zwischendurch (Gregg 2011: 54). Das Desinteresse des Managements, den Umfang der Arbeit einzuschränken, verbindet sich mit den Schwierigkeiten der Beschäftigten, ihrer Erwerbsarbeit Grenzen zu setzen. 
„Online technologies are a key factor in making today's jobs feel variously invasive, compelling, consuming, readily available, anxiety provoking, addictive ... and even the source of solace“ (Gregg 2011: 169). Damit spricht Gregg eine höchst ambivalente Wahrnehmung mobiler, digitaler Technik von einer Bedrohung einerseits bis zum Trost andererseits an, auf die auch Judy Wajcman hinweist.

Wajcman (2015) betont zum einen, dass die Kommunikationstechnologie E-Mail nicht nur eine Quelle, sondern auch ein Symbol für Stress in der Arbeit wurde. Zwar setzt tatsächlich die Menge der Nachrichten die Menschen unter Druck, diese schreiben den Stress jedoch oft der Technik E-Mail zu, während sie über die eigentlichen Ursachen nicht sprechen. Die Technik wird aber nicht nur als Quelle der Überlastung gesehen, sondern zugleich auch als Möglichkeit, Kontrolle über die Arbeit zu gewinnen und Belastungen zu reduzieren. Klarerweise ist hier nicht die Technik allein - etwa die Geschwindigkeit der Datenübertragung relevant, vielmehr sind die technischen Eigenschaften von E-Mail in Verbindung mit den Ängsten, die sie hervorrufen, und den Normen der Verwendung der Technik von wesentlicher Bedeutung. Mit Verwendungsnormen ist beispielsweise die Erwartung gemeint, rasch auf eine Nachricht zu antworten. Auch die zeitliche Verteilung der Verwendung über den Tag ist entscheidend für die Wirkungen. Gerade weil die Kommunikation zeitlich entkoppelt ist, im Gegensatz zum Telefonat also nicht auf beiden Seiten gleichzeitig erfolgt, häufen sich E-Mails im Eingangsordner. Zugleich besteht aber die Erwartung, dass auf E-Mails rasch reagiert wird. Zusammen führt das zu einer Ausweitung des Arbeitstages und dazu, dass die E-MailTechnologie zum Symbol für Stress werden kann, obwohl sie die Kommunikation einmal deutlich erleichtert hat (Wajcman 2015: 97) und tatsächlich auch die Vereinbarkeit zwischen Arbeit und außerbetrieblichen Verpflichtungen verbessern kann (Pfeiffer 2012). Auch in diesem Zusammenhang wäre es verfehlt, der Technik bestimmte - günstige oder ungünstige Wirkungen zuzuschreiben. Sie ist eher als ermöglichender Faktor zu sehen, der gesellschaftliche Trends, wie die Entgrenzung von Arbeit und Leben, verstärkt. Generell wird die Grenzziehung zunehmend dem Individuum abverlangt, wenn indirekte Steuerungsformen eine Selbstorganisation der Arbeit durch die Arbeitenden zulassen und auch verlangen und wenn kollektive Regelungen, etwa der Arbeitszeit, an Wirkung einbüßen. In entgrenzten Arbeitszusammenhängen wird es damit den Individuen überantwortet, die Voraussetzungen für die Reproduktion der Arbeitskraft sicherzustellen.

Erholung und soziale Einbindung stellen sich nicht mehr über kollektiv regulierte Schutzgrenzen des ,Privaten' her, sondern hängen von der Verhandlungsposition und Durchsetzungsstärke des Einzelnen ab. Die Person muss individuell vorsorgend tätig werden, muss Arbeit und Leben so austarieren, dass sich im Betrieb ,mithalten' lässt, die einmalige Ressource Arbeitskraft aber keinen Schaden nimmt. (Jürgens 2010: 502)

Insgesamt ist die Qualität der Arbeit nicht als Folge der Digitalisierung zu verstehen. Ebensowenig wie die technische Entwicklung einen notwendigen Weg nimmt, wie der Technikdeterminismus suggeriert, sind die Arbeitsfolgen auch schon durch die Technikgestaltung festgelegt. Doch diese Offenheit wird durch das neoliberale Programm der Veränderungen von Wirtschaft, Gesellschaft, Organisationen und Arbeit wieder geschlossen und impliziert eine einseitige auf Kapital- und Managementinteressen ausgerichtete Auslegung der Technikanwendung und der Arbeitsorganisation. Damit geht aber nicht notwendigerweise eine Degra- 
dierung der Arbeit einher, wenn diese auch empirisch neben der Aufwertung und relativen Autonomie sehr verbreitet zu beobachten ist.

\section{Die Plattformwirtschaft als Fluchtpunkt einer neoliberalen technologischen Revolution}

Niedrig entlohnte, diskontinuierliche und nicht dem Sozialversicherungsschutz unterliegende und in diesem Sinne prekäre Arbeit ist in vielen Ländern weit verbreitet und hat in den europäischen Wohlfahrtstaaten in den letzten Jahrzehnten zugenommen (Castel 2000; Standing 2011). Im Reinigungsgewerbe oder im Bauwesen haben diese Entwicklungen nichts mit Digitalisierung zu tun. In einem noch marginalen Bereich der Arbeitswelt besteht jedoch ein enger Zusammenhang zwischen neuen Technologien und prekärer Arbeit. Dieser wird als Plattformwirtschaft bezeichnet und stellt ein Musterbeispiel für das Zusammenwirken von neoliberaler Hegemonie und technologischer Entwicklung dar, die zusammengenommen die Arbeitsbeziehungen revolutionieren können. Im Kern handelt es sich um Internetplattformen, die zwischen Auftraggebern und Arbeitenden vermitteln. Ihre Ausgestaltung und Wirkung liegt meist irgendwo zwischen den traditionellen Gelben Seiten, über die man Handwerker/innen oder verschiedene Dienstleistungen finden konnte, einerseits, und Arbeitskräfteüberlassern andererseits, die Personal für kurzfristige Einsätze anbieten.

Grundsätzlich kann dabei zwischen Plattformen für internetgestützte digitale Arbeit, wie etwa Amazon Mechanical Turk, und Plattformen für ortsgebundene Arbeit, wie das bekannte Taxiservice Uber, unterschieden werden. Amazon Mechanical Turk (Lehdonvirta/Ernkvist 2011; Howcroft/Bergvall-Kåreborn 2014; Lehdonvirta 2016) ist ein Beispiel für Microwork, die Extremform der Standardisierung und Arbeitsteilung. Ähnlich der tayloristischen Zersplitterung der Arbeit bei manuellen Tätigkeiten kann digitale Arbeit in kleine Verrichtungen zerlegt werden. Microwork bezeichnet entsprechend die Erledigung kleinteiliger Aufgaben im Bereich der immateriellen Arbeit, die über Internetplattformen wie Amazon Mechanical Turk vermittelt wird. Auf diese Weise werden Aufgaben, wie die Kategorisierung von Bildern oder Videos oder die Eingabe der Daten von Einkaufsbelegen, als hochgradig repetitive Arbeit aufbereitet und pro einzelner, oft nur Sekunden benötigender Verrichtung jeweils mit wenigen Cent entlohnt. Amazon Mechanical Turk wurde entwickelt, als der Online-Handelskonzern Amazon eine Lösung für das Problem suchte, im eigenen großen Warenangebot ähnliche Produkte verschiedener Hersteller aufzufinden, um sie im Angebot verbinden zu können. Man dachte zuerst an eine technische Lösung mittels künstlicher Intelligenz und Bilderkennung. Doch nachdem das nicht gelang, entwarf man eine Plattform, um die Aufgabe, Waren zu vergleichen, an Menschen außerhalb des Unternehmens ausschreiben zu können. Die Lösung war dann also künstliche künstliche Intelligenz - das ist auch der Slogan, mit dem Amazon die Plattform anderen Auftraggebern für ähnliche Arbeiten anbietet. Mit Turk spielt man auf den Schachautomaten an, der 1769 Verwunderung hervorrief, weil eine als Türke gekleidete Puppe Schach spielen konnte, der allerdings in Wirklichkeit von einem versteckten Menschen geführt wurde (Gutknecht 2008: 43).

The devising and parceling out of these microtasks is arguably only the latest development in a lineage of work management that derives from Taylorism. Taskers are effectively de- 
skilled, dispersed, and deprived of any knowledge about the nature of the product to which their labor contributes. The coordinating manager, by contrast is in complete control of the labor process. (Ross 2013: 20-21)

Von den Plattformen für Microwork sind diejenigen Plattformen für ortsungebundene, immaterielle Arbeit zu unterscheiden, auf denen umfassendere Aufgaben ausgeschrieben werden, wie zum Beispiel projektbasierte Kreativarbeit. Letztere umfasst grafisches Design, Modedesign, Video, Audio, Webdesign, Spieleproduktion und vieles mehr. Die Vermittlung zwischen Auftraggeber/in und Auftragnehmer/in kann dabei auf zwei grundlegend unterschiedliche Arten erfolgen. Einerseits erfolgt die Vergabe von Aufträgen im Wettbewerbsverfahren (Pitch): Auf eine Einladung hin werden mehr oder weniger fertige Produkte eingereicht und nur der/die Gewinner/in wird bezahlt, während die anderen Teilnehmer/innen leer ausgehen. Die andere Form der Vergabe erfolgt durch ein Ausschreibungsverfahren, in dessen Rahmen Angebote (Preis, zeitlicher Rahmen) eingereicht werden und im Anschluss ein Zuschlag erteilt wird. Erst danach wird mit der Bearbeitung des Auftrags begonnen. Zusätzlich werden auf manchen Plattformen noch weitere Vertriebsmodelle angeboten, etwa Onlineshops, auf denen fertige Produkte (bspw. Logos, Videointros, Texte, Designs) zu Fixpreisen angeboten werden können. Die Arbeitenden präsentieren sich auf den Plattformen in standardisierten Profilen, die ihre Austauschbarkeit hervorheben (Huws 2016a).

Plattformen für ortsgebundene Arbeit bieten oder vermitteln eine Reihe von Dienstleistungen im Bereich des Transports, der Reinigung oder des Handwerks. So wird die Essenszustellung durch Fahrradboten von Firmen wie Deliveroo oder Foodora über Internetplattformen und Apps gesteuert. Auch hier ermöglichte es die Zugehörigkeit zur Plattformwirtschaft, sich teilweise außerhalb der für Arbeit geltenden Standards zu bewegen. Für die Arbeitenden, die ihre Rechte einzufordern versuchen, bedeutet dies ein Zurück an den Start in dem Sinne, dass sie kollektiven Widerstand organisieren müssen, um in den Genuss, der seit dem 19. Jahrhundert erkämpften und inzwischen weitgehend geltenden sozialen Rechte zu kommen. Bei Deliveroo in London wurde gestreikt, bei Foodora in Wien wurde ein Betriebsrat gegrün$\operatorname{det}$ (Herr 2017).

Die Beispiele aus der Plattformwirtschaft lassen sehr deutlich die Verbindung aus einer einseitigen Nutzung erweiterter Optionen zur Gestaltung der Arbeit, einer de facto Deregulierung und Re-Kommodifizierung der Arbeit und einem neuen spatial fix erkennen. Sie realisieren also ein neoliberales Programm, das sich in diesen Fällen offensichtlich durch den Technikdeterminismus, der die öffentliche Debatte dominiert, leichter umsetzen lässt. Demnach scheint es, als hätte die grundsätzlich als eigenlogisch gedachte technische Entwicklung eben Vor- und Nachteile. Im konkreten Fall der Plattformwirtschaft gehen die zerstörerischen Wirkungen davon aus, dass sie der Umgehung nationaler Regelungen des Konsumentenschutzes, des Arbeitsrechts, der Mindestlöhne und der Beiträge zur Sozialversicherung dienen (Schmidt 2017: 9). Diese technologiebasierten Geschäftsmodelle ziehen gerade deshalb Investoren und Investorinnen an, weil sie als disruptive Innovationen dargestellt werden können, was nichts anderes bedeutet, als dass sie geltende Spielregeln unterlaufen. Die Plattformbetreiber/innen stellen sich vielfach als Technologieunternehmen dar, benutzen neue digitale Techniken aber eher als Trojanisches Pferd. Denn zwischen einer traditionellen Funkzentrale eines Taxiunternehmens und der App von Uber besteht im Hinblick auf die Vermittlung von 
Fahrten kein qualitativer Unterschied. Dargestellt als neues Geschäftsmodell wird dennoch versucht, sowohl die Regeln des Gewerbes als auch die arbeitsrechtlichen Bestimmungen zu umgehen, indem die Fahrer/innen als Selbstständige behandelt werden.

Bei dieser Form der Auslagerung von Arbeit wird generell ein Arbeitsverhältnis, also eine rechtlich geregelte Beziehung zwischen Arbeitgeber/innen und Arbeitnehmer/innen, vermieden - sowohl zwischen Auftraggeber/innen und Ausführenden, als auch zwischen Plattform und Arbeitenden. Die Betreiber/innen der Internet-Plattformen treten vielmehr als Vermittler/innen auf: Sie bringen Arbeitskräfte, die ihre Dienste über die Plattform anbieten, mit Auftraggeber/innen zusammen, die für ein bestimmtes Projekt oder eine Tätigkeit eine Arbeitskraft suchen. Und sie bieten umfangreiche Funktionen für die Kommunikation zwischen Auftraggeber/innen und -nehmer/innen, für die Abwicklung der Bezahlung und für die Bewertung der Arbeitsleistung an. Im Vereinigten Königreich haben die Gerichte dieser Strategie bereits eine Grenze gesetzt: Bereits in der zweiten Instanz wurde inzwischen festgestellt, dass Uber-Fahrer/innen Arbeitnehmer/innen sind und ihnen der gesetzliche Mindestlohn gebührt.

Im Bereich der ortsungebundenen, immateriellen Arbeit vermeiden die Plattformbetreiber/innen im Crowdsourcing ebenfalls das Entstehen von regulären Arbeitsverhältnissen im Dreieck zwischen Auftraggebenden, Vermittelnden und Arbeitenden. Sie nutzen die Unklarheit darüber, ob es sich bei den Arbeitenden um Arbeitnehmer/innen handelt, die insbesondere bei einer Betrachtung nach Vertragsbeziehungen entsteht (Risak 2016). Damit wenden sie die Anwendung von Schutzbestimmungen im Hinblick auf die Arbeitszeit und das Einkommen, aber auch sozialrechtliche Absicherungen ab. Es wird also eine de facto Deregulierung der Arbeit durch die Vermeidung von Anstellungsverhältnissen und die Einrichtung abhängiger Selbstständigkeit im Rahmen einer "triangularisierten Beschäftigung“ (Helfen 2014) erreicht.

Innerhalb der Dreieckskonstellation wird sowohl von Plattformbetreiber/innen als Kund/innen aber erheblicher Druck auf die Arbeitenden ausgeübt, der auf ein Abhängigkeitsverhältnis schließen lässt. Ein Beispiel dafür sind die Bewertungen der Arbeit durch die Auftraggebenden mittels eines standardisierten Punkte- oder Sternesystems. Die Möglichkeit der Bewertung beeinflusst das Verhalten der Auftragnehmenden, welche für das Erlangen von Aufträgen auf positive Beurteilungen angewiesen sind. Dies führt insbesondere bei nicht gut am Markt etablierten Arbeitenden zur Bereitschaft, auch unbezahlt zu arbeiten, etwa wenn die Kund/innen Änderungen verlangen (Schörpf et al. 2017). Kurze Reaktionszeiten, ständige Erreichbarkeit und die Einhaltung kurzer Fristen sind auch für die Bewertung durch die Plattform auf Basis von Datenauswertungen entscheidende Kriterien. Zusammengenommen wird es oft unmöglich, Nachtarbeit zu vermeiden, das Wochenende arbeitsfrei zu halten oder auf Urlaub zu fahren. Mit diesen Problemen haben die Alleinselbstständigen verschiedenster Branchen abseits der Plattformwirtschaft schon lange zu kämpfen. Der Unterschied besteht darin, dass nicht nur die Kunden, sondern auch die Plattformbetreiber/innen die Crowdworkers unter Druck setzen (Schörpf et al. 2017). Die einseitig von den Plattformbetreiber/innen gestalteten Merkmale und Prozesse der Plattform sehen Möglichkeiten nicht nur zur Bewertung, sondern auch zur Überwachung der Arbeitenden vor und unterbinden in der Regel die Kommunikation mit anderen Crowdworkers. 
Neben einer de facto Deregulierung und der einseitigen Bestimmung über Technik- und Organisationsgestaltung kommt an diesem Beispiel auch sehr deutlich der spatial fix zum Tragen. Die Grundidee des Crowdsourcing ist ja gerade die Chance, Arbeit an unbestimmt viele unabhängig von deren geografischem Standort auszuschreiben. Damit erfolgt also zugleich mit der Auslagerung eine räumliche Verlagerung von Arbeit. Grundsätzlich kann durch dieses Crowdsourcing ein globaler Arbeitsmarkt entstehen, bei dem ein globales Cybertariat als industrielle Reservearmee genutzt wird (Huws 2003). Eine tatsächlich gleichmäßige Globalisierung ist bisher jedoch noch nicht zu erkennen. So sind Sprachgebiete vielfach nach wie vor relevant, wodurch außer im angelsächsischen Raum die Internetplattformen primär im nationalstaatlichen Rahmen aktiv zu sein scheinen (Mandl et al. 2015).

Die Plattformwirtschaft ist nicht in erster Linie dem Internet und damit verbundenen neuen technischen Möglichkeiten geschuldet. Sie ist vielmehr die Spitze des Eisbergs einer umfassenden Umgestaltung der Arbeitswelt im finanzialisierten Kapitalismus (Thompson 2003; Deutschmann 2008; Windolf 2013). Damit ist die Dominanz der Finanzmarktakteure im globalen Kapitalismus gemeint, die zu starkem Druck zur Erzielung hoher Renditen und zur Steigerung der Aktienkurse geführt hat und sich auch auf das Machtverhältnis zwischen Kapital und Arbeit auswirkt. Zugleich drückt sich die Finanzialisierung in der Möglichkeit aus, extrem hohe Kapitalausstattung für Unternehmen mit neuen Geschäftsmodellen zu erreichen, die eine kreative Zerstörung von Branchen versprechen und damit spätere hohe Gewinne. Für die Arbeitenden ist in diesem Zusammenhang entscheidend, dass die Stabilität der Gewinneinkommen und die Pflege der Börsekurse, die dem Shareholder Value-Prinzip gemäß oberste Priorität in den Strategien der Unternehmen haben, die Möglichkeit der Anpassung der Lohnkosten an die Auftragslage und damit eine Flexibilisierung der Arbeit voraussetzt. Zum Ideal wird die Arbeit auf Abruf, auf das mit verschiedenen Formen der Arbeitszeitflexibilisierung, mit dem Einsatz von Leiharbeitskräften und mit der Auslagerung von Arbeit an andere Firmen hingearbeitet wird. Die Internetplattformen treiben dieses Prinzip auf die Spitze, indem Arbeitskraft für einzelne Aufgaben zur Verfügung gestellt wird, die von wenigen Minuten, wie im Fall von Microwork, bis zu Stunden oder Tagen dauern können. Über die Erledigung der Arbeit hinaus besteht keinerlei Verpflichtung der Auftraggeber/innen den Arbeitenden gegenüber. Eine gern zitierte Aussage eines Plattformmanagers bringt das auf den Punkt:

Before the Internet, it would be really difficult to find someone, sit them down for ten minutes and get them to work for you, and then fire them after those ten minutes. But with technology, you can actually find them, pay them the tiny amount of money, and then get rid of them when you don't need them anymore. (CEO der Firma Crowdflower zitiert in The Nation, 24. Februar 2014)

Offensichtlich ist die Technologie für solche Arbeitsbeziehungen nützlich. Aber sie ist nicht die Ursache dafür, dass es diese gibt. Die Ursache ist vielmehr das Streben nach höheren Gewinnen auf Seiten der Unternehmen und der „stumme(n) Zwang der ökonomischen Verhältnisse" (Marx 1962: 764) auf Seiten der Arbeitenden. Und es ist ein normativer Rahmen nötig, der es rechtlich erlaubt und moralisch nicht als anstößig definiert, Menschen nur für kurze Aufgaben zu beschäftigen und keinerlei Verantwortung für ihr Gesamteinkommen und damit für ihr Überleben zu übernehmen. Es müssen also TINA - hier als Kürzel für die neoliberale 
Hegemonie verwendet - und spezifische Verwendungen technologischer Potenziale zusammenkommen, um die beschriebenen Effekte zu erzielen.

Auf Crowdworking trifft damit in besonderem Maße zu, was Castells über die Netzwerkgesellschaft insgesamt behauptet hat: „Under the conditions of the network society, capital is globally coordinated, labor is individualized“" (Castells 2001: 507). Die Arbeitenden sind geografisch verstreut und sozial in hohem Maße vereinzelt, da die Plattformen in der Regel keine Möglichkeiten zur horizontalen Kommunikation bieten. Im Fall von Microwork ist auch keine Kommunikation zwischen Auftraggebern bzw. Auftraggeberinnen und Arbeitenden vorgesehen, weil der Arbeitsprozess wie automatisiert ablaufen soll und das Lesen eines E-Mails höhere Kosten verursachen würde als ein gesamter Arbeitsauftrag (Irani 2015; Lehdonvirta 2016).

Doch dieselbe Technologie, die es erlaubt, vereinzelte Arbeitende in einen übergreifenden Arbeitsprozess einzubinden, bietet grundsätzlich auch den Arbeitenden die Chance, sich zu vernetzen und ihre Interessen zu befördern. So gründete ein für Amazon Mechanical Turk Arbeitender im Jahr 2008 eine Onlinecommunity, auf der sich die Turker austauschen konnten. Durch solche Initiativen entstehen soziale Räume, in denen gemeinsame Interessen sowie berufliche und Klassenidentitäten zum Ausdruck gebracht werden (Lehdonvirta 2016: 75). Ausnahmeerscheinungen dürften gemeinnützige Plattformen - wie CloudFactory in Nepal sein, die von sich aus einen Austausch unter den online Arbeitenden fördern, indem sie diese anregen, sich zu virtuellen Teams von jeweils fünf Personen zusammenzuschließen, eine Facebook-Gruppe zu gründen und sich auch persönlich zu treffen (Lehdonvirta 2016: 70).

Die Plattform Turkopticon ist ebenfalls ein Beispiel für die Aneignung der Technologie im Interesse der Crowdworker. Sie dient der Beurteilung der Auftraggeber/innen durch die Turker - diese drehen den Spieß also im Vergleich zur üblichen Bewertung der Arbeitenden um. Dadurch ist es den Arbeitenden möglich, die Wahrscheinlichkeit zu reduzieren, an betrügerische Auftraggeber/innen zu kommen, nicht bezahlt zu werden oder das Arbeitsergebnis unbegründet zurückgewiesen zu bekommen. Dies sind nämlich häufige Beschwerden der Turker. Die Gründer/innen von Turkopticon geben an, dass die Plattform über 55.000 registrierte User und 20.000 Besuche pro Monat habe und dass seit 2009 ca. 287.000 Bewertungen über 42.000 Auftraggeber/innen gesammelt wurden (Irani/Silberman 2016).

Der Umfang der sogenannten Plattformökonomie und ihr Entwicklungspotenzial sind nach wie vor schwer abzuschätzen. Dies und die oben diskutierten Aspekte der Digitalisierung werfen die Frage auf, ob die neuerliche Diskussion über eine technologische Revolution so wie frühere wieder abflauen wird, ohne dass der Gesellschaft die Arbeit ausgegangen oder das reguläre Arbeitsverhältnis samt sozialer Absicherung der Arbeitenden ausgehebelt sein werden. Es gibt viele Gründe anzunehmen, dass es diesmal anders ausgehen könnte.

In my opinion, this time round we really are facing a sea-change in work organisation. This is not so much because an entirely new phenomenon is emerging (although it undoubtedly is) but precisely because of the cumulative impact of all the other changes that commentators have been noting over the past forty years. Sector by sector, occupation by occupation, company by company, disparate trends have reached critical mass, converging to produce a kind of snowball effect in which each change induces others, with the potential for generating an avalanche. (Huws 2016b) 
Mit diesen unterschiedlichen Trends meint Huws die verstärkte Standardisierung von Arbeit und Organisation, die Modularisierung von Organisationen und Geschäftsprozessen, die eine stärkere Aufteilung und räumliche Verlagerung erlaubt, sowie die Informalisierung von Arbeit durch Auslagerung an Selbstständige. Auch darin sind die drei eingangs dargestellten Dimensionen des Zusammenwirkens der neoliberalen Hegemonie und des technologischen Umbruchs, nämlich die einseitige Gestaltung von Technik und Arbeit, der spatial fix und die de facto Deregulierung deutlich zu erkennen. Am Beispiel der Plattformwirtschaft zeigt sich auch die gegenseitige Verstärkung dieser Trends, insofern räumliche Verlagerung und Streuung die Informalisierung der Arbeit erleichtert oder die einseitige Gestaltung sowohl auf räumliche Aspekte als auch auf die Umgehung der Regulierung von Arbeit abzielt.

\section{Zusammenfassung}

In den aktuellen Debatten über Industrie 4.0 und Digitalisierung herrscht ein Technikdeterminismus insofern vor, als die technische Entwicklung einer Eigenlogik folgend und damit als festgelegt und notwendig dargestellt wird. Von den Unternehmen und den Arbeitskräften wird eine möglichst rasche Anpassung an einen Trend verlangt, der als nicht beeinflussbar erscheint und von dem umfangreiche Auswirkungen auf Produkt- und Arbeitsmärkte ausgehen. Staatliche und politische Akteure sollen jedoch den technologischen Wandel und die Anwendung der Technologien fördern, damit die Unternehmen des jeweiligen Landes Vorreiter der Entwicklung sein und so ihre Wettbewerbsfähigkeit stärken können. Kaum wird thematisiert, dass die technologische Entwicklung und die Gestaltung der Technik gesellschaftlich bestimmt und einzelnen Gruppeninteressen verpflichtet ist. Die vielfachen Optionen, Entscheidungsmöglichkeiten und Scheidewege werden in einen linear gedachten technischen Fortschritt umgedeutet, bei dem man entweder vorne mit dabei ist oder aber ins Hintertreffen gerät. Das Paradox und die beachtliche Leistung der Debatte bestehen also darin, die enorme Ausweitung an Gestaltungsmöglichkeiten in einen einseitigen Anpassungszwang umzudeuten.

Deutlich ist zu erkennen, wie sehr die Debatte ein Kind ihrer Zeit ist. Die Humanisierung der Arbeit klingt zwar in Slogans an, wie in jenem, wonach der Mensch dank Industrie 4.0 frei werde für kreative Tätigkeiten. Doch wird die Verbesserung der Arbeitsbedingungen nicht zu einem expliziten Ziel, mit dem die Orientierung an Wettbewerbsfähigkeit auch nur ansatzweise ergänzt würde. In früheren Automationsdiskussionen, etwa in den 1980er Jahren, war noch von mannlosen Nachtschichten die Rede gewesen, wodurch die gesundheitsgefährdende Nachtarbeit eingeschränkt werden könne. Damals hatte das politische Ziel der Humanisierung der Arbeit offensichtlich noch auf die Debatten über Technik und Automation eingewirkt. Heute veranlasst die neoliberale Hegemonie zu einem weiter verengten Zugang (Pfeiffer 2017). Von technischen Entwicklungen ermöglichte neue Optionen werden bloß noch zur Steigerung der Wettbewerbsfähigkeit und darüber hinaus für neue Geschäftsmodelle genutzt, mit denen insbesondere aus der Umgehung sozialstaatlicher Regelungen und sozialer Rechte der Arbeitenden Gewinn geschlagen wird. Legitimiert wird das mit dem disruptiven Charakter der Technologie von morgen, die eben nicht in die Regulierungen von gestern eingesperrt werden dürfe. 
Karl Marx hat in Erfindungen und Technik unter anderem ein „Kriegsmittel des Kapitals“ gegen Arbeiteraufstände gesehen (Marx 1962: 459). Auch heute gelingt es einzelnen Unternehmen enorme Kapitalinvestitionen mit dem Versprechen zu lukrieren, ganze Märkte aufzumischen und bisherige Regeln für die Arbeit zu umgehen. Es ist nicht zu leugnen, dass die Technologie und die Art und Weise, in der sie in gesellschaftspolitischen Diskursen gerahmt wird, erheblich zur „Entbettung“ im Polanyi’schen Sinn (Polanyi 1977) der neuen Arbeitsformen und -beziehungen beigetragen haben. Die sogenannte Plattformwirtschaft mit ihren teilweise extrem hohen Unternehmensbewertungen ist ein gutes Beispiel dafür, wie das neoliberale Einheitsdenken die disruptiven Innovationen ermöglicht, die gerne der Technologie zugeschrieben werden. Entsprechend präsentieren sich Unternehmen wie Uber oder Amazon als Technologieunternehmen, und wird der Technikdeterminismus zur Legitimierung des Wandels herangezogen. Die neuen Geschäftsmodelle werden zunächst ohne Rücksicht auf sozialstaatliche und arbeitsrechtliche Regelungen in die Realität umgesetzt. Den Arbeitenden in diesen Firmen kommt dann die Aufgabe zu, ihre sozialen Rechte über kollektives Handeln und Gerichtsverfahren neuerlich zu erkämpfen.

Der Technikdeterminismus spielt neben der hier TINA genannten neoliberalen Hegemonie deshalb eine so wichtige Rolle, weil die Einsicht in die gesellschaftliche Gestaltung der Technik und in die Rolle der Technik als Ermöglicherin (und nicht als Ursache) unmittelbar die Frage nach sich zieht, welche Gruppen in der Gesellschaft über die Entwicklungsrichtung bestimmen und welche alternativen Wege denn noch möglich wären. Zwar wurden Internet und Digitalisierung vielfach auch als Potenziale einer umfassenden Demokratisierung und eines Wandels der Wirtschaft in Richtung einer Sharing Economy gesehen. Damit wurde der digitalen Revolution einer von TINA nicht vorgesehener Weg gewiesen. Doch inzwischen ist an der umfassenden Kommerzialisierung des Internet und der immateriellen Güter deutlich geworden, wie stark der Finanzmarktkapitalismus die Entwicklungen der Gesellschaft nach wie vor bestimmt. Der den Proponenten und Proponentinnen der Sharing Economy eigene Technikdeterminismus könnte diesen (vorläufigen) Ausgang mit erklären. Denn auch in diesen Szenarien wurde der Technik die Rolle der treibenden Kraft zuerkannt, während es doch starker gesellschaftlicher Kräfte gebraucht hätte, um eine solche Umwälzung der Gesellschaft in die Wege zu leiten.

In diesem Beitrag ging es darum aufzuzeigen, dass die Verschränkung der als linear und notwendig gedeuteten Technikentwicklung im Bereich der Informations- und Kommunikationstechnologien mit einem neoliberalen gesellschaftspolitischen Programm sich insbesondere in den drei Dimensionen der einseitig kapital- und managementorientierten Gestaltung und Anwendung der Technik, im Versuch der räumlichen Lösung betriebswirtschaftlicher Probleme in einem liberalisierten Finanzmarktkapitalismus und in einer Informalisierung der Arbeit als de facto Deregulierung fassen lässt. Der Entwicklung der Arbeit in der Plattformökonomie wird zu Recht große Aufmerksamkeit geschenkt. Schließlich kommen bei ihr alle diese Aspekte gemeinsam zum Tragen. Von der gesellschaftlichen Reaktion auf diese nur angeblich technologische Revolution könnte die Zukunft der Erwerbsarbeit abhängen. 


\section{Literatur}

Altmann, Norbert, Manfred Deiß, Volker Döhl und Dieter Sauer (1986). Ein „Neuer Rationalisierungstyp“ - neue Anforderungen an die Industriesoziologie. Soziale Welt, 37(2/3), 191-207.

Arntz, Melanie, Terry Gregory und Ulrich Ziehran (2016). The risk of automation for jobs in OECD countries: a comparative analysis. OECD Social, Employment and Migration Working Papers No. 189, Paris: OECD.

Bain, Peter, Aileen Watson, Gareth Mulvey, Phil Taylor und Gregor Gall (2002). Taylorism, targets and the pursuit of quantity and quality by call centre management. New Technology, Work and Employment, 17(3), 170-185.

Boes, Andreas und Tobias Kämpf (2011). Global verteilte Kopfarbeit. Offshoring und der Wandel der Arbeitsbeziehungen. Berlin: edition sigma.

Böhle, Fritz (2010). Arbeit als Handeln. In: Böhle, Fritz, Gerd-Günther Voß und Günther Wachtler (Hg.). Handbuch Arbeitssoziologie. Wiesbaden: VS Verlag für Sozialwissenschaften, 151-176.

Butterwegge, Christoph, Rudolf Hickel und Ralf Ptak (1998). Sozialstaat und neoliberale Hegemonie. Standortnationalismus als Gefahr für die Demokratie. Berlin: Elefanten Press.

Carter, Bob, Andrew Danford, Debra Howcroft, Helen Richardson, Andrew Smith und Phil Taylor (2011). 'All they lack is a chain': lean and the new performance management in the British civil service. New Technology, Work and Employment, 26(2), 83-97.

Castel, Robert (2000). Die Metamorphosen der sozialen Frage. Eine Chronik der Lohnarbeit. Konstanz: UKV Universitätsverlag.

Castells, Manuel (2001). Das Informationszeitalter. Band 1: Der Aufstieg der Netzwerkgesellschaft. Opladen: Leske + Budrich.

Deutschmann, Christoph (2008). Kapitalistische Dynamik. Eine gesellschaftstheoretische Perspektive. Wiesbaden: Verlag für Sozialwissenschaften.

Flecker, Jörg und Pamela Meil (2010). Organisational restructuring and emerging service value chains - implications for work and employment. Work, Employment and Society, 24(4), 1-19.

Flecker, Jörg und Annika Schönauer (2016). The Production of 'Placelessness': Digital Service Work in Global Value Chains. In: Flecker, Jörg (Hg.). Space, Place and Global Digital Work. London: Palgrave Macmillan, 11-30.

Frade, Carlos und Isabelle Darmon (2005). New Modes of Business Organization and Precarious Employment: Towards the Recommodification of Labour? Journal of European Social Policy, 15(2), 107-121.

Frey, Carl Benedikt und Michael A. Osborne (2013). The future of employment: how susceptible are jobs to computerisation. Oxford: University of Oxford.

Goel, Vindu (2017). IBM Now Has More Employees in India Than in the U.S. New York Times, 28.09.2017. Internet-Site. Zugriff am 28.05.2018 auf https://www.nytimes.com/2017/09/28/technology/ibm-india.html

Gregg, Melissa (2011). Work's Intimacy. Malden, Mass.: Polity Press. 
Gutknecht, Christoph (2008). Von Treppenwitz bis Sauregurkenzeit: die verrücktesten Wörter im Deutschen. München: C.K. Beck.

Haidinger, Bettina (2012). On the move in Global Delivery Chains: Labour Relations and Working Conditions in the Parcel Delivery Industries of Austria, Germany, the Czech Republic and Hungary. SODIPER Synthesis Report. Wien: FORBA.

Harvey, David (2001). Spaces of capital - towards a critical geography. New York: Routledge.

Helfen, Markus (2014). Netzwerkförmige Tertiarisierung und triangularisierte Beschäftigung: Braucht es eine interorganisationale Personalpolitik? Managementforschung, 24, 171-206.

Herr, Benjamin (2017). Riding in the Gig Economy. AK Working Paper. Wien: AK.

Holtgrewe, Ulrike, Thomas Riesenecker-Caba und Jörg Flecker (2016). „Industrie 4.0“ Eine arbeitssoziologische Einschätzung. FORBA im Auftrag der Kammer für Arbeiter und Angestellte für Wien.

Howcroft, Debra und Brigitta Bergvall-Kåreborn (2014). Amazon Mechanical Turk and the Commodification of Labour. New Technology, Work \& Employment, 29(3), 213-223.

Howcroft, Debra und Helen Richardson (2012). The Back Office Goes Global: Exploring Connections and Contradictions in Shared Service Centres. Work, Employment \& Society 26(1): 111-127.

Huws, Ursula (2003). The making of the cybertariat - virtual work in a real world. New York: Monthly Review Press.

Huws, Ursula (2016a). Eingeloggt. Kurswechsel 2/2016, 7-12.

Huws, Ursula (2016b). Platform labour: Sharing Economy or Virtual Wild West? Journal for a Progressive Economy, January 2016, 24-27.

Irani, Lilly (2015). The cultural work of microwork. New Media and Society, 17(5), 720-739.

Jürgens, Kerstin (2010). Arbeit und Leben. In: Böhle, Fritz, Gerd-Günther Voß und Günther Wachtler (Hg.). Handbuch Arbeitssoziologie. Wiesbaden: VS Verlag, 483-510.

Kern, Horst und Michael Schumann (1984). Das Ende der Arbeitsteilung? Rationalisierung in der industriellen Produktion: Bestandsaufnahme, Trendbestimmung. München: Beck.

Lehdonvirta, Vili und Mirko Ernkvist (2011). Knowledge Map of the Virtual Economy. Washington DC: World Bank.

Lehdonvirta, Vili (2016). Algorithms That Divide and Unite: Delocalization, Identity, and Collective Action in 'Microwork'. In: Flecker, J. (Hg.). Space, Place and Global Digital Work. London: Palgrave Macmillan, 53-80.

MacKenzie, Donald A. und Judy Wajcman (Hg.) (1985). The Social Shaping of Technology. Milton Keynes: Open University Press.

Lutz, Burkhart (1979): Das Verhältnis von Technik, Bildung und Arbeit als Problem politischen Handelns. Berufsbildung in Wissenschaft und Praxis 8(5): 8-11.

Mandl, Irene, Sara Riso und Elias Gerogiannis (2015). New forms of employment. Dublin: Eurofound.

Marx, Karl (1962). Das Kapital. Band 1. Wien: Globus Verlag. 
Morgan, Glenn (2005). Understanding Multinational Corporations. In: Ackroyd, S., R. Batt, P. Thompson, und P.S. Tolbert (Hg.). The Oxford Handbook of Work \& Organization. Oxford: Oxford University Press, 554-576.

Nagl, Wolfgang, Gerlinde Titelbach und Katarina Valkova (2017). Digitalisierung der Arbeit: Substituierbarkeit von Berufen im Zuge der Automatisierung durch Industrie 4.0. Wien: Projektbericht IHS.

Noble, David F. (1979). Social choice in machine design: the case of automatically controlled machine tools. In: Zimbalist, A. (Hg.). Case studies on the labour process. New York: Monthly Review Press, 18-50.

OECD (2017). OECD Economic Surveys: Switzerland 2017. Paris: OECD Publishing.

Pfeiffer, Sabine (2010). Technisierung von Arbeit. In: Böhle, Fritz, Gerd-Günther Voß und Günther Wachtler (Hg.). Handbuch Arbeitssoziologie. Wiesbaden: VS, Verlag für Sozialwissenschaften, 231362 .

Pfeiffer, Sabine (2012). Technologische Grundlagen der Entgrenzung: Chancen und Risiken. In: Badura, Bernhard, Antje Ducki, Helmut Schröder, Joachim Klose und Markus Meyer (Hg.). FehlzeitenReport 2012. Gesundheit in der flexiblen Arbeitswelt: Chancen nutzen - Risiken minimieren. Berlin, Heidelberg: Springer, 15-21.

Pfeiffer, Sabine (2017). Industrie 4.0 in the Making - Discourse Patterns and the Rise of Digital Despotism. In: Briken, Kendra, Shiona Chillas, Martin Krzywdzinski und Abigail Marks (Hg.). The New Digital Workplace. How Technologies Revolutionise Work. Palgrave Macmillan: Basingstoke, 21-41.

Polanyi, Karl (1977). The Great Transformation. Politische und ökonomische Ursprünge von Gesellschaften und Wirtschaftssystemen. Wien: Europaverlag.

Promotorengruppe Kommunikation der Forschungsunion Wirtschaft-Wissenschaft (2013). Umsetzungsempfehlungen für das Zukunftsprojekt Industrie 4.0. Abschlussbericht des Arbeitskreises Industrie 4.0. Frankfurt a. Main.

Ramioul, Monique und Geert van Hootegem (2015). Relocation, the Restructuring of the Labour Process and Job Quality. In: Drahokoupil, Jan (Hg.). The Outsourcing Challenge: Organizing Workers across Fragmented Production Networks. Brussels: European Trade Union Institute (ETUI), 91115.

Risak, Martin (2016). What's Law got to do with it? (Arbeits-)Rechtliche Aspekte plattformbasierten Arbeitens. Kurswechsel 2/2016, 32-41.

Ritzer, George, Paul Dean und Nathan Jurgenson (2012). The Coming of Age of the Prosumer. American Behavioral Scientist, 56(4), 379-398.

Rosa, Hartmut (2005). Beschleunigung. Die Veränderung der Zeitstrukturen in der Moderne. Frankfurt/Main: Suhrkamp.

Ross, Andrew (2013). In search of the lost paycheck. In: Scholz, T. (Hg.). Digital labor: the Internet as playground and factory. New York: Routledge, 13-32.

Rubery, Jill (2010). Institutionalizing the Employment Relationship. In: Morgan, G., J.L. Campbell, C. Crouch, O.K. Pedersen und R. Whitley (Hg.). The Oxford Handbook of Comparative Institutional Analysis. Oxford: Oxford University Press, 497-525. 
Schmidt, Florian A. (2017). Digital Labour Markets in the Platform Economy - Mapping the Political Challenges of Crowd Work and Gig Work. Bonn: Friedrich Ebert Stiftung.

Schörpf, Ph., J. Flecker, A. Schönauer und H. Eichmann (2017). Triangular love-hate: management and control in creative crowdworking. New Technology, Work and Employment, 32(1), 43-58.

Spath, Dieter (Hg.) (2013). Produktionsarbeit der Zukunft - Industrie 4.0. Stuttgart: Fraunhofer Verlag.

Sproll, Martina (2016). Missing Links in Service Value Chain Analysis - The Case of Call Centres in the Brazilian Banking Sector. In: Flecker, Jörg (Hg.). Space, Place and Global Digital Work. London: Palgrave Macmillan, 105-126.

Standing, Guy (2011). The Precariat - the new dangerous class. London: Bloomsbury.

Thompson, Paul (2003). Disconnected capitalism: or why employers can't keep their side of the bargain. Work, Employment and Society, 17(2), 359-378.

Wajcman, Judy (2015). Pressed for time - The Acceleration of Life in Digital Capitalism. Chicago: The University of Chicago Press.

Weil, David (2014). The fissured workplace. Cambridge, Mass.: Harvard University Press.

Williams, Robin und David Edge (1996). The social shaping of technology. Research Policy 25(6), 865899.

Will-Zocholl, M. (2016). New Topologies of Work: Informatisation, Virtualisation and Globalisation in Automotive Engineering. In: Flecker, Jörg (Hg.). Space, Place and Global Digital Work. London: Palgrave, 31-52.

Windelband, Lars und Georg Spöttl (2012). Diffusion von Technologien in der Facharbeit und deren Konsequenzen für die Qualifizierung am Beispiel des „Internet der Dinge“. In: Faßhauer, Uwe, Bärbel Fürstenau und Eveline Wuttke (Hg.). Berufs- und wirtschaftspädagogische Analysen. Aktuelle Forschungen zur beruflichen Bildung. Opladen: Verlag Barbara Budrich, 205-219.

Windelband, Lars und Georg Spöttl (2012). Diffusion von Technologien in der Facharbeit und deren Konsequenzen für die Qualifizierung am Beispiel des „Internet der Dinge“. In: Faßhauer, Uwe, Bärbel Fürstenau und Eveline Wuttke (Hg.). Berufs- und wirtschaftspädagogische Analysen. Aktuelle Forschungen zur beruflichen Bildung. Opladen: Verlag Barbara Budrich, 205-219.

Windolf, Paul (2013). Finanzmarkt-Kapitalismus. In: Hirsch-Kreinsen, Hartmut und Heiner Minssen (Hg.). Lexikon der Arbeits- und Industriesoziologie. Berlin: edition sigma, 215-222.

Wolter, Marc Ingo, Anke Mönnig, Markus Hummel, Christian Schneemann, Enzo Weber, Gerd Zika, Tobias Maier, Caroline Neuber-Pohl und Robert Helmrich (2015). Industrie 4.0 und die Folgen für Arbeitsmarkt und Wirtschaft. Szenariorechnungen im Rahmen der BIBB-IAB-Qualifikations- und Berufsfeldprojektionen. IAB-Forschungsbericht Nr. 8, Nürnberg: IAB. 